\title{
Dynamic force measurements of avidin-biotin and streptavdin-biotin interactions using AFM
}

\author{
Marzena de Odrowąż Piramowicz ${ }^{1}$, Paweł $\mathrm{Czuba}^{2}$, Marta Targosz², \\ Květoslava Burda ${ }^{1,2 \varpi}$ and Marek Szymoński ${ }^{2}$ \\ ${ }^{1}$ Institute of Nuclear Physics, Polish Academy of Sciences, Kraków, Poland; ${ }^{2}$ Institute of Physisc, Jagiellonian

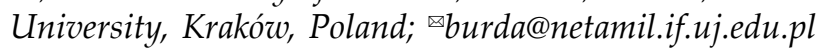

Received: 16 February, 2005; revised: 27 April, 2005; accepted: 30 September, 2005

available on-line: 12 January, 2006

\begin{abstract}
Using atomic force microscopy (AFM) we performed dynamic force measurements of the adhesive forces in two model systems: avidin-biotin and streptavidin-biotin. In our experiments we used glutaraldehyde for immobilization of (strept)avidin on the tip and biotin on the sample surface. Such interface layers are more rigid than those usually reported in the literature for AFM studies, when (strept)avidin is coupled with biotinylated bovine albumin and biotin with agarose polymers. We determined the dependence of the rupture forces of avidin-biotin and streptavidin-biotin bonds in the range $300-9600 \mathrm{pN} / \mathrm{s}$. The slope of a semilogarithmic plot of this relation changes at about $1700 \mathrm{pN} / \mathrm{s}$. The existence of two different regimes indicates the presence of two activation barriers of these complexes during the dissociation process. The dissociation rates and activation energy barriers, calculated from the Bell model, for the avidin-biotin and streptavidin-biotin interactions are similar to each other for loading rates $>1700 \mathrm{pN} / \mathrm{s}$ but they are different from each other for loading rates $<1700 \mathrm{pN} / \mathrm{s}$. In the latter case, the dissociation rates show a higher stability of the avidin-biotin complex than the streptavidin-biotin complex due to a larger outer activation barrier of $0.8 k_{B} T$. The bond-rupture force is about $20 \mathrm{pN}$ higher for the avidin-biotin pair than for the streptavidin-biotin pair for loading rates $<1700 \mathrm{pN} / \mathrm{s}$. These two experimental observations are in agreement with the known structural differences between the biotin binding pocket of avidin and of streptavidin.
\end{abstract}

Keywords: avidin, streptavidin, biotin, atomic force microscopy, rupture force, dissociation rate, loading rate

Living organisms are complex highly ordered systems. Physico-chemical interactions between their arranged components regulate the living processes on a molecular level. The equilibrium between the repulsive and attractive forces determines the minimum of the potential net energy for a given complex of molecules. It characterizes the stability of such a ligand-receptor system at certain environmental conditions. Attractive forces, which bring the molecules together, are divided into strong and weak interactions. Strong ionic and covalent bonds have fixed angles and lengths and often store chemical energy.
Energies of hetero- and monopolar interactions are of the order of $100-1000 \mathrm{~kJ} / \mathrm{mol}$. Energies of weak attractions (van der Waals forces, ion-dipole forces, hydrogen bonds) are an order of magnitude smaller. Although these interactions are relatively weak, they are important because of their numbers.

Weak bonds are responsible for intermolecular and intramolecular attractions of biological molecules and therefore they are of great importance (Fersht, 1985; Dammer et al., 1996; Bongrand, 1999). For example, recognition of substrate by enzyme, antigen by antibody or neurotransmitter by neurore-

Abbreviations: AFM, atomic force microscopy; APTES-(3-aminopropyl)triethoxysilane; BFP, biomembrane force probe; $F_{\mathrm{i}}$ bond rupture force; $k$, dissociation rate in the presence of an external force; $k_{\mathrm{B}^{\prime}}$ Boltzmann constant; $k_{\mathrm{C}^{\prime}}$ spring constant of cantilever; $k_{\text {eff }}$ effective spring constatnt; $k_{\mathrm{s}^{\prime}}$ spring constant of elastic substrates and covers; $k_{0}$, dissociation rate in the absence of an external force; $L_{\mathrm{r}^{\prime}}$ force loading rate; ML, microlever; OT, optical tweezers; PBS, phosphate-buffered saline; $r_{0}$, width of potential energy well; $T$, absolute temperature; UL, ultralever. 
ceptor rely on weak, non-covalent binding forces. The architecture of the participants and their flexibility are important factors in this process. Investigations of the reversibility and specificity of ligand-receptor interactions are essential to fully understand the formation and dissociation of individual bonds in more complex biosystems.

There are several biophysical methods allowing direct measurements of ligand-receptor interactions, for example: optical tweezers (OT) (Askin \& Dziedzic, 1987), biomembrane force probe (BFP) (Evans et al., 1995), magnetic torsion device (Guttenberg et al., 2000) or atomic force microscopy (AFM) (Binning et al., 1986). Using AFM equipped with a liquid-cell one can measure short range forces in biological systems under physiological conditions. Typical detected forces are in the range from a few $\mathrm{pN}$ to several $\mu \mathrm{N}$. Moreover, this method allows one to measure molecular binding forces between a small number of various ligand and receptor molecules because of the size of the AFM cantilever. One of the interacting particles is immobilized on the cantilever and the other on a surface. The adhesion is probed with force-distance curves. The rupture (unbinding) force, being a measure of the interaction between the ligand and the receptor, is detected upon withdrawal of the cantilever from the surface.

In this paper we present dynamic studies of the unbinding force in two model systems for protein-ligand interaction systems: avidin-biotin and streptavidin-biotin. Chicken avidin and bacterial streptavidin proteins are tetrameric molecules having a molecular mass of about $60 \mathrm{kDa}$ and 66 $\mathrm{kDa}$, respectively. Both proteins contain four identical subunits, each with one binding site for biotin. These proteins are so interesting because of their extremely high binding affinity for biotin (vitamin $\mathrm{H}$ ) (Green, 1975; Lindqvist \& Schneider, 1996). In the avidin-biotin and streptavidin-biotin complexes the dissociation constants of the range of $10^{-15} \mathrm{M}^{-1}$ are much higher than the affinities detected for very stable antigen-antibody complexes $\left(10^{-6}-10^{-9} \mathrm{M}^{-1}\right)$. Van der Waals forces between biotin and the aromatic side chains of the binding residues of the proteins, an extreme network of hydrogen bonds between the protein pockets and the ligand, and the rearrangement of L3,4 loops of the proteins upon biotin biding are the recognized interactions mainly contributing to the extremely tight biotin bonds with avidin and streptavidin (Freitag et al., 1997; Livnah et al., 1993; Grubmüller et al., 1996). These specific interactions have been utilized in different applications in medicine and biotechnology (Bayer \& Wichek, 1990; Shetters, 1996; Wilchek \& Bayer, 1999; Akahara \& Saga, 1999).

Measurements of the unbinding forces between (strept)avidin and biotin (or modified biotin forms) using AFM dynamic force spectroscopy have already been performed by several groups (Florin et al., 1994; Allen et al., 1996; Merkel et al., 1999; Wong et al., 1999; Yuan et al., 2000; Lo et al., 2001; Zhang \& Moy, 2003). However, there are some discrepancies in the observed relations between the measured bond rupture forces and the force loading rates (retraction rates) for the analyzed complexes. One suspects that experimental conditions, namely the multiple links between the adsorption surface and the proteins as well as the flexibility of the intermediate layers may have influenced the observed ligand-receptor unbinding forces. In order to verify this hypothesis we studied the avidin-biotin and streptavidin-biotin interactions in a modified experimental protocol applying AFM.

We used the Bell model for describing the relation between the rupture forces of these two complexes and the applied force loading rates. Our results are in agreement with Evans et al. (1995) predictions that one should observe multiple sections on the graphs of rupture forces versus the logarithm of loading rates for a (strept)avidin-biotin complex. The slope of each section corresponds to a different energy state of the system. The observed relations between the unbinding forces of the avidin-biotin and streptavidin-biotin complexes are consistent with our knowledge regarding the structural differences between their biotin-binding pockets.

\section{MATERIALS AND METHODS}

AFM force measurements. We applied an atomic force spectrometer AFM (Park Scientific Instruments) equipped with a liquid cell for adhesive force measurements. One of AFM components is a cantilever tip mounted on a small arm and deflected by external forces. Deflection of the cantilever is monitored by a laser beam reflected from an upper surface of the cantilever and detected in a 4-segment photodetector. The difference between the photocurrent of the top and bottom segments gives information about forces acting between the tip and a sample.

Two kinds of cantilevers were used to obtain a wide range of loading rates. Silicon nitride unsharpened cantilevers, microlevers, with a spring constant $k_{c}=0.01 \mathrm{~N} / \mathrm{m}$ (ML06C type, MLCT-NONM, ThermoMicroscopes, Sunnyvale, CA, USA) and silicon cantilevers, ultralevers, with a spring constant $k_{c}=0.4 \mathrm{~N} / \mathrm{m}$ (UL06B type, ULCT-AUNM, ThermoMicroscopes, Sunnyvale, CA, USA) were applied for loading rates from 300 to $4000 \mathrm{pN} / \mathrm{s}$ and from 4000 to $9600 \mathrm{pN} / \mathrm{s}$, respectively. We independently checked the spring constants of the cantilevers using Sader method (Sader et al., 1999) based on the meas- 
urements of the resonant frequency. The estimated spring constants agreed with the nominal values within 3-8\%. All force scan measurements were performed in phosphate-buffered saline (PBS: $20 \mathrm{mM}$ $\mathrm{PO}_{4}^{3-}, 150 \mathrm{mM} \mathrm{NaCl}, \mathrm{pH}=7.2$ ) at room temperature.

Preparation of biosensors and sample surface. Cantilever tips coated with avidin or streptavidin are called biosensors. Such coated tips were then brought into contact with a substrate covered with immobilized biotin. The cantilevers and substrates were functionalized with (strept)avidin and biotin, respectively, according to the method described by Lukham and Smith (1998). The procedure is shown in Fig. 1.

A tip, after washing in acetone, was incubated in 10\% APTES (3-amino-propyltriethoxysilane) for two hours in order to create amino groups $\left(-\mathrm{NH}_{2}\right)$ on its surface. Then, the tip was kept in $2.5 \%$ glutaraldehyde for $20 \mathrm{~min}$. Finally, it was coupled with avidin (avidin from egg white, $0.17 \mathrm{mg}$ protein $/ \mathrm{ml}$ PBS, Sigma-Aldrich) or with streptavidin (Streptavidin-C-Phycocyanin, $0.17 \mathrm{mg}$ protein/ml PBS, Fluka, Sigma-Aldrich) during a 10-min incubation. Glutaraldehyde mediated (strept)avidin binding via its aldehyde groups. Functionalized tips were stored at $4^{\circ} \mathrm{C}$ until needed.

The sample surface was coated with biotin (D-Biotin, $1 \mu \mathrm{g} / 1 \mathrm{ml}$ PBS, Sigma-Aldrich,) in a similar way as described for the tips. We could omit the first stage of the procedure presented in Fig. 1 because we used glass plates covered with poly-Llysine, which provided exposed amino groups. The substrate surface was incubated in $2.5 \%$ glutaraldehyde and then biotin was immobilized on it.

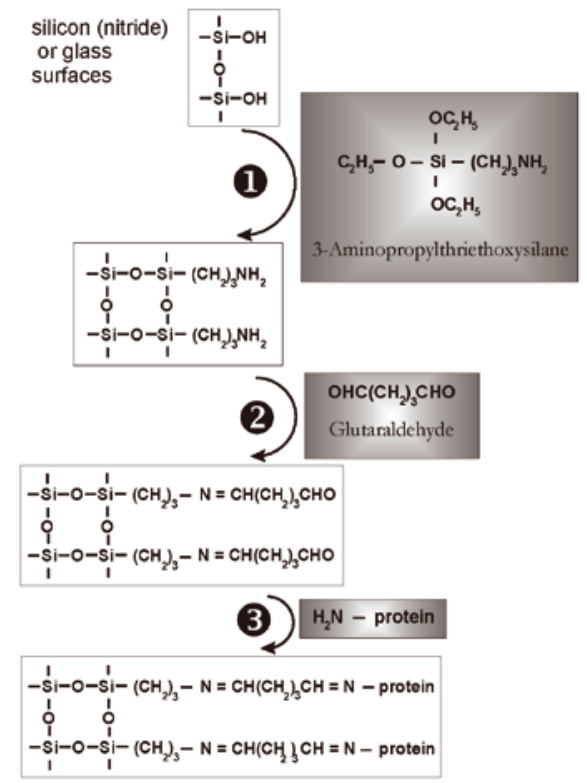

Figure 1. Scheme of (strept)avidin-functionalized AFM tip preparation.

Substrate silanization with APTES (1), glutaraldehyde as a cross-linking mediator (2), protein immobilization (3).

\section{RESULTS}

Molecular binding forces between biotin and avidin/streptavidin molecules were studied by AFM. The force measurements were carried out with a substrate surface coated with biotin moving towards the (strept)avidin functionalized tip. During the approach-retract cycles one can observe unbinding events as sharp jumps of the cantilever. The length of the jump is taken as a measure of the interaction between the ligand and the acceptor. An example of a force-displacement curve for the streptavidinbiotin system is shown in Fig. 2. The upper curve corresponds to the approach and the lower one to the retraction of the cantilever tip. There are three visible transitions in the force measurement presented in Fig. 2. The rupture forces assigned as F1, $F 2$ and F3 correspond to the applied force needed to break one, three or two bonds in a streptavidinbiotin complex, assuming that the $F 1$ force is the unbinding force of a single streptavidin-biotin pair for the force loading rate of $3900 \mathrm{pN} / \mathrm{s}$ under our experimental conditions. One can find that $F 1 \approx 255$ $\mathrm{pN}, F 2 \approx 3 \times F 1 \approx 765 \mathrm{pN}$ and $F 3 \approx 2 \times F 1 \approx 510 \mathrm{pN}$. We checked the specificity of the interactions adding free biotin, which abolished the adhesion forces. In about $95 \%$ of cases for which the adhesion has been detected (about $60 \%$ of all trials), the retract traces of the force measurements exhibited from one to several transitions, whose magnitudes corresponded to a single rupture force of the avidin/streptavidin-biotin pair. This means that under our experimental conditions with a rigid interface layer (APTES + glutaraldehyde) connecting the ligand (receptor) to the tip (subgrade), we observed mainly independent molecule adhesion due to sequential breakage of bonds in the (strept)avidin-biotin pairs. Elastic and flexible

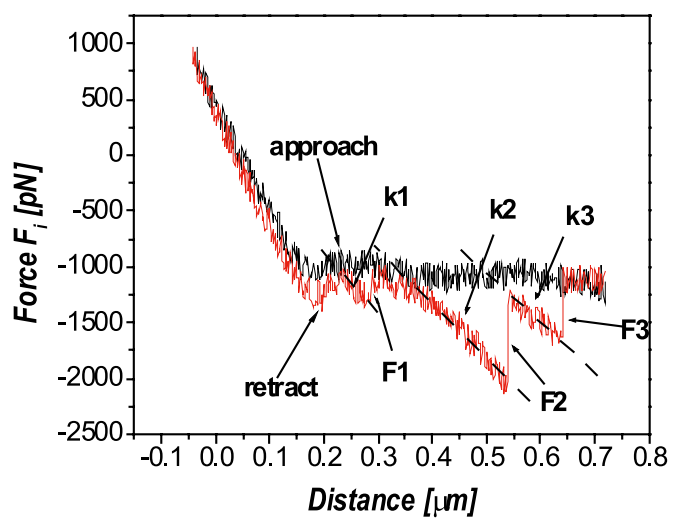

Figure 2. Force-displacement curves of the streptavidinbiotin interaction at the loading rate of $3900 \mathrm{pN} / \mathrm{s}$.

Approach and retraction traces are indicated. F1, F2 and F3 are rupture forces corresponding to a single, triple and double bond of the streptavidin-biotin complex, respectively. Effective spring constant $k_{\text {eff }}$ of the streptavidinfunctionalized tip can be estimated from the slope of the force vs. displacement curve as shown in this figure (dotted lines $\left.k_{1}, k_{2}, k_{3}\right)$. 
intermediate cross linkers, such as agarose beads binding biotin and biotinylated bovine serum albumin attaching (strept)avidin, result in a higher probability of cooperative adhesion (Wong et al., 1999). However, the probability of multiple bond ruptures also increases with an increasing force loading rate (Fig. 2). Representative histograms of rupture forces derived from about 100 force measurements of avidin-biotin and streptavidin-biotin interactions at similar rates of cantilever retractions are shown in Figs. 3A and 3B, respectively. The average unbinding force of a single biotin-streptavidin or biotin-avidin pair was determined from such a force histogram at a given cantilever retraction velocity. A single Gaussian function was fitted to the force histograms (Fig. 3). We carried out adhesion strength measurements of the chosen ligand-protein systems under various scan rates using cantilevers of different spring constants. In this way we achieved variation in the force loading rate $L_{r}$. It is well known that the measured disrupting force increases with increasing loading rate (Evans \& Ritchie, 1997; Merkel et al., 1999). Moreover, chemical fixation of the ligand and of the acceptor influences the system compliance (Lo et al., 2001). This means that the effective spring constant $k_{\text {eff }}$ which should be considered in the calculations of the force loading rate, differs from that of the cantilever applied, $k_{c}$. Taking into account the spring constant of the elastic background and the
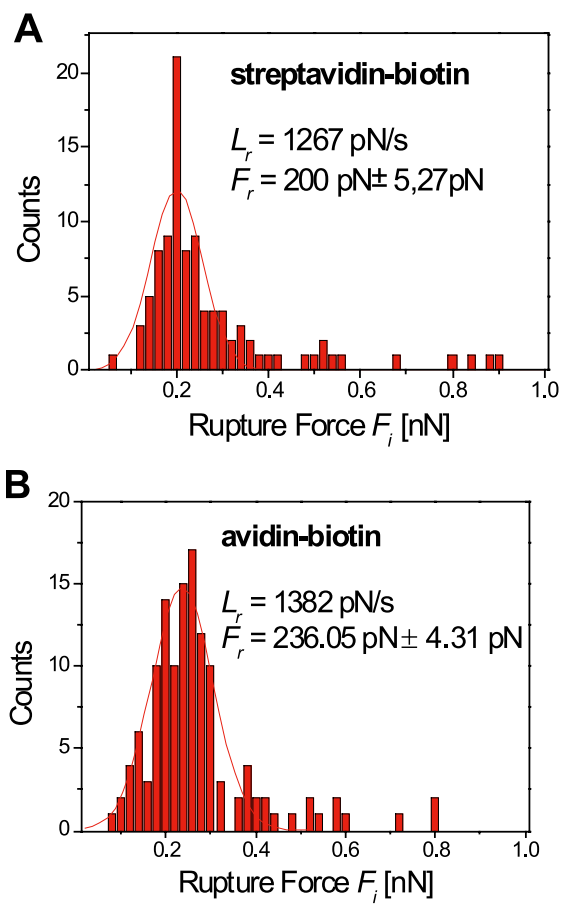

Figure 3. Force histograms of about 100 measurements for streptavidin-biotin (A) and avidin-biotin (B) interactions with fitted Gaussian functions.

The average unbinding force for streptavidin-biotin is 200 $\mathrm{pN}$ at the force loading rate of $1267 \mathrm{pN} / \mathrm{s}$ (A) and for avidin-biotin it is $236 \mathrm{pN}$ at the force loading rate of 1382 $\mathrm{pN} / \mathrm{s}(\mathrm{B})$. attached probes, $k_{s^{\prime}}$ one can calculate the effective spring constant from the relation: $1 / k_{\text {eff }}=1 / k_{s}+1 / k_{c}$. The elasticity of the substrates may cause a decrease of the system spring constant and in addition an increase of the rupture force. Therefore, it is important to estimate the effective spring constant. Usually it is not possible to calculate it directly because of the incomplete knowledge on the molecular linkages contributing to $k_{s}$. The spring constant can be, however, obtained from the average slope of the force vs. displacement of the retraction traces (see Fig. 2). The measured effective spring constants in our experiments were in the range of $2-12 \mathrm{mN} / \mathrm{m}$. The curves representing the dependence of force measurements on the loading rates in the range from $300 \mathrm{pN} / \mathrm{s}$ to $9600 \mathrm{pN} / \mathrm{s}$ for the avidin-biotin and sterptavidin-biotin complexes are shown in Fig. 4.

\section{DISCUSSION}

Dynamic force measurements of unbinding forces in two investigated systems avidin-biotin and streptavidin-biotin, are summarized in Fig. 4. One clearly sees two regimes of the bond rupture dependence on the logarithm of the force loading rate $(l n L r)$ in the range from 300 to $9600 \mathrm{pN} / \mathrm{s}$. This observation is consistent with theoretical predictions. Bell's theory (Bell, 1978) predicts that the ligand-acceptor dissociation rate constant $k$ depends exponentially on the externally applied force $F$ :

$$
k(F)=k_{0}(0) \exp \left(\frac{F r_{0}}{k_{B} T}\right)
$$

where $k_{B}$ is the Boltzmann constant, $T$ is absolute temperature, $r_{0}$ is the distance between the bound and the transition state projected along the direction of applied force, $k_{0}$ is the dissociation rate constant

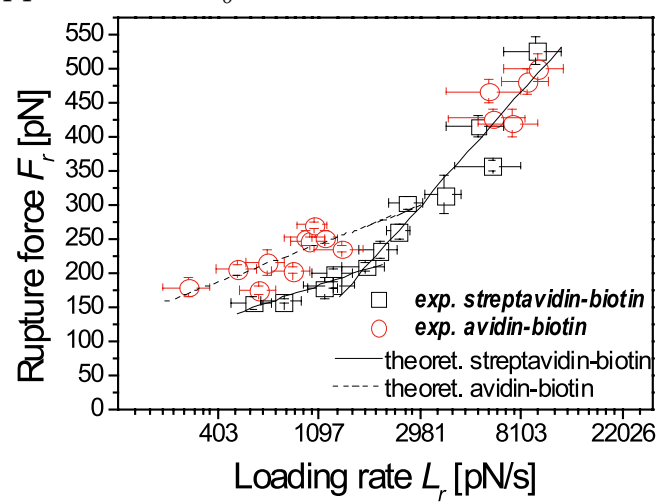

Figure 4. Loading rate dependence of the rupture force for the streptavidin-biotin interactions ( $\square$ ) and the avidin-biotin interactions (o).

Symbols represent experimental data. Lines are theoretical fits applying the Bell model for the two force regimes: a solid line for the streptavidin-biotin complex and a dotted line for the avidin-biotin complex. 
in the absence of an external force. Later Evans and Ritche (1997) using the Bell model and the Kramer theory on reaction kinetics (Kramers, 1940) derived a formula for the dependence of the unbinding force $F_{i}$ on the force loading rate $L_{r}$ :

$F i=\frac{k_{B} T}{r_{0}} \ln \left(\frac{L_{r} r_{0}}{k_{0} k_{B} T}\right)$

This exponential dependence of the inter- and intramolecular unbinding forces on the loading rate has already been demonstrated (Rief et al., 1997; Fritz et al., 1998; Oberhauser et al., 1998). Plotting the rupture force $F_{\mathrm{i}}$ vs. $\ln \left(L_{\mathrm{r}}\right)$ one can estimate the distance between the energy potential minimum and the energy barrier maximum, $r_{0}$, for a given regime of applied external forces from the slope of the fitted line. The dissociation rate constant, $k_{0^{\prime}}$ can be calculated from the intercept with the abscissa at zero force. According to the formula given in Eqn. 2, the parameters characterizing the binding states are expressed as follows:

$$
\begin{aligned}
& r_{0}=\frac{k_{B} T}{\text { slope }} \\
& k_{0}=\frac{L_{r}\left(F_{i}=0\right) r_{0}}{k_{B} T}
\end{aligned}
$$

The dissociation rates and the width parameters of the energy wells obtained from the linear fits to our experimental data of rupture forces for avidin/streptavidin-biotin (Fig. 4) are collected in Table 1 . In the region of high loading rates, above $1700 \mathrm{pN} / \mathrm{s}$, a potential barrier at a distance of about $0.024 \mathrm{~nm}$ from the center of the binding pocket dominates and it has a similar width for both avidin-biotin and streptavidin-biotin interactions. The dissociation rates are also equal within the error bars for both systems in the regime of loading rates above $1700 \mathrm{pN} / \mathrm{s}$ and are about $3 \mathrm{~s}^{-1}$. However, for lower force loading rates, that is from $300 \mathrm{pN} / \mathrm{s}$ to about $1700 \mathrm{pN} / \mathrm{s}$, the measured force slope of avidin-biotin differs from that of streptavidin-biotin. The width of the potential barrier is slightly lower for the avidin-biotin complex $(0.07 \mathrm{~nm})$ than for the streptavidin-biotin complex $(0.08 \mathrm{~nm})$ and this difference is statistically significant. The dissociation rate of the avidin-biotin pair $\left(0.25 \mathrm{~s}^{-1}\right)$ is about two times lower than of the streptavidin-biotin pair $(0.56$ $\mathrm{s}^{-1}$ ), but both dissociation rates for lower rupture forces are lower than $k_{0}$ obtained for the region of high unbinding forces. This means that the avidin/ streptavidin-biotin complexes are more stable for force loading rates $<1700 \mathrm{pN} / \mathrm{s}$. The relative stability of the avidin-biotin and streptavidin-biotin pairs can be estimated from the ratio of the dissociation rates $\left(k_{\text {Oav }} / k_{\text {Ostr }}\right)$ of these two systems (see Table 1$)$. Our data shows that the avidin-biotin interaction is stronger than the streptavidin-biotin one due to the higher outer activation energy barrier of 0.86 $k_{B} T$ (at room temperature it is about $2.1 \mathrm{~kJ} / \mathrm{mol}$ ). The inner activation energy barriers for these systems are similar to each other. The same conclusions we obtained from the estimated bond lifetimes of the complexes in the presence of a disrupting force, by dividing two standard deviations of the unbinding force by the force loading rate. The bond lifetimes for both systems are roughly constant and similar to each other at large force loading rates $>4000 \mathrm{pN} / \mathrm{s}$ and are about $5.0 \pm 1.7 \mathrm{~ms}$. In the range of low force loading rates from $300 \mathrm{pN} / \mathrm{s}$ to $1700 \mathrm{pN} / \mathrm{s}$ the bond lifetimes vary: they decrease with the increasing force loading rate from about $80 \mathrm{~ms}$ in the case of the avidin-biotin complex and from about $40 \mathrm{~ms}$ in the case of the streptavidin-biotin complex for 300 $\mathrm{pN} / \mathrm{s}$ to about $10 \mathrm{~ms}$ in both cases for $1700 \mathrm{pN} / \mathrm{s}$.

The multiple transitions between avidin/ streptavidin-biotin intermediate states formed during the unbinding process observed in our experiments are in agreement with the predictions of molecular dynamic simulations, which have shown several potential energy wells for avidin-biotin and streptavidin-biotin interactions (Grubmüller et al., 1996; Izrailev et al., 1997). Thus, the slope of each section in Fig. 4 represents the characteristic force loading rate scale for a specific energy barrier (an energy well) that dominates in the region of the unbinding pathway. We observed similar regimes of the force loading rate for the state transitions of avidin/streptavidin-biotin interactions as Yuan et al. (2000) did but different relations between the subsequent states of avidin and streptavidin. Our data shows the existence of equilibrium binding states similar for avidin-biotin and streptavidinbiotin for higher force loading rates but different for lower loading rates, in agreement with Merkel et al. (1999), albeit at different ranges of the force loading rates. Using the data of the biomembrane force probe (BFP) experiments (Merkel et al., 1999) one can show that the inner activation energy barriers are similar to each other but the outer activation energy barrier for the avidin-biotin complex is higher by about $9.4 k_{B} T$ (at room temperature it is about $23 \mathrm{~kJ} / \mathrm{mol}$ ). Thus, only the outer activation energy is responsible for the stronger interaction between avidin and biotin, as was observed in our case. Yuan et al. (2000) applying AFM have also found that the avidin-biotin system is more stable than the streptavidin-biotin one but as a result of an increase of both activation energy barriers inner and outer ones. In their case the contribution of the inner energy barrier is about three times higher than the outer energy barrier, being equal to 10.95 
Table 1. Bell model parameters obtained from the dependence of $F_{i}$ on $\ln \left(L_{r}\right)$, where $F_{i}-$ the unbinding force and $L_{r}$ - the force loading rate

\begin{tabular}{|c|c|c|c|c|c|}
\hline Range of $L_{r}[\mathrm{pN} / \mathrm{s}]$ & Slope $[\mathrm{pN}]$ & $r_{0}[\mathrm{~nm}]$ & Intercept $[\mathrm{pN}]$ & $k_{0}\left[\mathrm{~s}^{-1}\right]$ & References \\
\hline \multicolumn{6}{|l|}{ Streptavidin-biotin } \\
\hline $300-1700$ & $50.1 \pm 9.4$ & $0.081 \pm 0.002$ & $-167.7 \pm 65.9$ & $0.56 \pm 0.46$ & \\
\hline $1700-9600$ & $166.3 \pm 20.9$ & $0.024 \pm 0.003$ & $-1032.3 \pm 171.5$ & $2.98 \pm 2.61$ & \\
\hline $100-1000$ & & 0.49 & & $1.67 \times 10^{-5}$ & Yuan et al., 2000 \\
\hline $1000-5000$ & & 0.05 & & 2.09 & Yuan et al., 2000 \\
\hline $0.05-10000$ & & 0.5 & & ${ }^{1} 6.14 \times 10^{-5}$ & Merkel et al.,1999 \\
\hline $10000-60000$ & & 0.12 & & ${ }^{1} 2.9$ & Merkel et al.,1999 \\
\hline \multicolumn{6}{|l|}{ Avidin-biotin } \\
\hline $300-1700$ & $55.6 \pm 15.5$ & $0.073 \pm 0.002$ & $-146.1 \pm 103.2$ & $0.25 \pm 0.18$ & \\
\hline $1700-9600$ & $168.6 \pm 14.8$ & $0.024 \pm 0.002$ & $-1047.6 \pm 125.2$ & $2.97 \pm 2.71$ & \\
\hline $100-1000$ & & 0.53 & & $6.45 \times 10^{-6}$ & Yuan et al., 2000 \\
\hline $100-5000$ & & 0.2 & & 0.08 & Yuan et al., 2000 \\
\hline $0.05-30$ & 1.4 & 3.0 & & & Merkel et al.,1999 \\
\hline 30-10000 & $13-14$ & 0.3 & & ${ }^{1} 0.74$ & Merkel et al.,1999 \\
\hline $10000-60000$ & & 0.12 & & ${ }^{1} 2.9$ & Merkel et al.,1999 \\
\hline
\end{tabular}

${ }^{1}$ The dissociation rates are estimated by us from extrapolation of the rupture force to zero for subsequent regimes (Fig. $3 \mathrm{~b}$, Merkel et al. 1999).

$k_{B} T$ (at room temperature it is $\sim 24 \mathrm{~kJ} / \mathrm{mol}$ ) and 3.3 $k_{B} T$ (at room temperature it is about $8.2 \mathrm{~kJ} / \mathrm{mol}$ ), respectively.

The higher stability of the avidin-biotin than the streptavidin-biotin system is in accordance with the chemical and structural differences between the biotin binding pockets of avidin and of streptavidin. There are three extra hydrogen bonds and hydrophobic interactions in avidin (Weber et al., 1989; Livnah et al., 1993). In addition, in avidin the L3,4 loop is longer and can bind biotin more tightly (Weber et al., 1989; Livnah et al., 1993; Freitag et al., 1997). It has been recently proved that hydrophobic interactions (Li et al., 2003) and structural complementarity (Morttilo et al., 2003) are the most important factors determining the strength of non-covalent bonds. In our experiments the avidin-biotin complex is more stable than the streptavidin-biotin one for the range of low loading rates. The similar stabilities of the avidin-biotin and streptavidin-biotin interactions at the regime of higher loading rates are due to the diminishing importance of structural factors in the interactions under such experimental conditions.

One may expect that the bond rupture forces should increase with the increasing strength of ligand-receptor interactions. Indeed, in our experiments, for the force loading rates of 300-1700 pN/s the unbinding forces of the avidin-biotin pair are higher by about $20 \mathrm{pN}$ than for the streptavidin-biotin pair. Surprisingly, weaker rupture forces are reported for the avidin-biotin complex than for the streptavidin-biotin one usually (Wong et al., 1999; Merkel et al., 1999; Yuan et al., 2000). This effect may result form less rigid interface layers anchoring the ligand-receptor systems. In the case of AFM measurements (Wong et al., 1999, Yuan et al., 2000) (strept)avidin was coupled to the cantilever tip via biotinylated bovine serum albumin (BSA) and biotin was bound to agarose polymers. For the biomembrane force probe (BFP) (Merkel et al., 1999) biotinylated polyethylene glycol (PEG)-polymers were used as the subgrade for (strept)avidin molecules whereas PEG-biotin polymers were linked to a red blood cell.

From a comparison of the parameters describing the dynamic behavior of the unbinding forces collected in Table 1 and the measured bond-rupture forces (Merkel et al., 1999; Yuan et al., 2000; this work) it is obvious that interpretation of the measured rupture forces is a difficult and complex task. One has to take into account possible modifications of the compliance of the ligand-receptor system due to the experimental protocol because the primary source of errors in this analysis comes from the uncertainty of the spring constant value of the whole system including the cross-linkers. Therefore, in or- 
der to understand the relation between the rupture forces measured by AFM and the real non-covalent bonds in the studied biocomplexes, more systematic investigations of ligand-receptor interactions on model systems are necessary.

Summarizing, the results presented in this paper show that the rupture forces measured for the avidin-biotin and streptavidin-biotin complexes depend on the rate of the applied external force. We observed transitions between intermediate states formed during the unbinding processes of both studied systems. Using the Bell model we were able to derive the width of the energetic barriers and dissociation constants for two different regimes of the dissociation process studied for the avidin- and streptavidin-biotin systems. The results are explained in the frame of the known structural and hydrophobic differences between the biotin binding pockets of avidin and streptavidin. The arrangement of the biotin binding sites is important in the regime of the low force loading rates but its influence on the detected rupture forces diminishes in the regime of the higher force loading rates. This indicates that the enhanced outer activation energy barrier for the avidin-biotin complex is responsible for its higher stabilization. The difference between the outer potential barriers is estimated to be about $0.86 k_{B} T$. The rupture forces stronger by about $20 \mathrm{pN}$ for the avidin-biotin pair than for the streptavidin-biotin pair observed at force loading rates below $1700 \mathrm{pN} / \mathrm{s}$ are in agreement with this conclusion. This observation that the avidin-biotin complex is more stable than the streptavidin-biotin one is independently supported by the bond lifetimes evaluated for the studied systems at different ranges of applied force loading rates.

\section{Acknowledgements}

This work was partially supported by the grants $3 \mathrm{~T} 11 \mathrm{E} 03326$ and 2 P04A 04427 from the Committee for Scientific Research (KBN, 2004-2007), Poland.

\section{REFERENCES}

Allen S, Davies J, Dawkes AC, Davies MC, Edwards JC, Parker MC, Roberts CJ, Sefton J, Tendler SJB, Williams PM (1996) In situ observation of streptavidin-biotin binding on an immunoassay well surface using an atomic force microscope. FEBS Lett 390: 161-164.

Ashkin A, Dziedzic JM (1987) Optical trapping and manipulation of viruses and bacteria. Science 235: 1517-1520.

Bayer EA, Wilchek M (1990) Application of avidin \pm biotin technology to affinity-based separations. J Chromatog 510: 3-11.

Bell GI (1978) Models for the specific adhesion of cells to cells. Science 200: 618-627.
Binnig G, Quate CF, Gerber CH (1986) Atomic force microscopy. Phys Rev Lett 56: 930-933.

Bongrand P (1999) Ligand-receptor interactions. Rep Prog Phys 62: 921-968.

Dammer U, Hegner M, Anselmetti D, Wagner P, Dreier M, Huber W, Güntherodt HJ (1996) Specific antigen/antibody interactions measured by force microscopy. Biophys J 70: 2437-2441.

Evans E, Ritchie K, Merkel R (1995) Sensitive force technique to probe molecular adhesion and structural linkages at biological interfaces. Biophys J 68: 2580-2587.

Evans E, Ritchie K (1997) Dynamic strength of molecular adhesion bonds. Biophys J 72: 1541-1555.

Fersht A (1985) Enzyme Structure and Mechanism. 2nd edn, WH Freeman and Company, New York.

Florin E-L, Moy VT, Gaub HE (1994) Adhesion forces between individual ligand-receptor pairs. Science 264: 415-417.

Freitag S, Le Trong I, Klumb L, Stayton PS, Stenkamp RE (1997) Structural studies of the streptavidin binding loop. Protein Sci 6: 1157-1166.

Fritz JA, Katopodis G, Kolbinger F, Anselmetti D (1998) Force mediated kinetics of single P-selectin/ligand complexes observed by atomic force microscopy. Proc Natl Acad Sci USA 95: 12283-12288.

Green NM (1975) Avidin. Adv Protein Chem 29: 85-133.

Grubmüller H, Heymann B, Tavan P (1996) Ligand binding: molecular mechanics calculation of the streptavidin-biotin rupture force. Science 271: 997-999.

Guttenberg Z, Bausch AR, Bin Hu, Bruinsma R, Morader L, Sackman E (2000) Measuring ligand-receptor unbinding forces with magnetic beads: molecular average. Langmuir 16: 8984-8993.

Izrailev S, Stepaniants S, Balsera M, Oono Y, Schulten K (1997) Molecular dynamics study of unbinding of the avidin-biotin complex. Biophys J 72: 1568-1581.

Kramers HA (1940) Brownian motion in a field of force and the diffusion model of chemical reactions. Physica 7: 284-302.

Li Y, Li H, Yang F, Smith-Gill SJ, Mariuzza RA (2003) XRay snapshots of the maturation of an antibody response to a protein antigen. Nat Struct Biol 6: 482-488.

Linvah O, Bayer EA, Wilchek M, Sussman JL (1993) Threedimential structure of avidin and the avidin-biotin complex. Proc Natl Acad Sci USA 90: 5076-5080.

Lo YS, Zhu Y, Beebe TP (2001) Loading-rate dependence of individual ligand-receptor bond-rupture forces studied by atomic force microscopy. Langmuir 17: 3741-3748.

Luckham PF, Smith K (1998) Direct measurements of the recognition forces between protein and membrane receptors. Faraday Disc 111: 307-320.

Marttila AT, Hytönen VP, Laitinen OH, Bayer EA, Wilchek M, Kuomaa MS (2003) Mutation of the Tyr33 residue of chicken avidin: functional and structural consequences. Biochim J 369: 249-254.

Merkel R, Nassoy P, Leung A, Ritchie K, Evans E (1999) Energy landscapes of receptor-ligand bonds explored with dynamic force spectroscopy. Nature 397: 50-53.

Oberhauser AF, Marszalek PE, Erickson HP, Fernandez JM (1998) The molecular elasticity of the extracellular matrix tenascin. Nature 393: 181-185.

Rief M, Oesterhelt F, Heymann B, Gaub HE (1997) Single molecule force spectroscopy on polysaccharides by atomic force microscopy. Science 275: 1295-1297.

Sader JE, Chon JWM, Mulvaney P (1999) Calibration of rectangular atomic force microscope cantilevers. Rev Sci Instrum 70: 3967-3969. 
Sakahara H, Saga T (1999) Avidin-biotin system for delivery of diagnostic agents. Adv Drug Deliv Rev 37: 89-101.

Schetters H (1999) Avidin and streptavidin in clinical diagnostics. Biomol Eng 17: 73-78.

Weber PC, Ohlendorf DH, Wendoloski JJ, Salemme FR (1989) Structural origins of high affinity biotin binding to streptavidin. Science 243: 85-88.

Wilchek M, Bayer EA (1999) Foreword and introduction to the book (Strept)avidin-biotin system. Biomol Eng 16: $1-4$.
Wong J, Chilkoti A, Moy VT (1999) Direct force measurements of streptavidin-biotin interaction. Biomol Eng 16: $45-55$.

Yuan Ch, Chen A, Kolb P, Moy VT (2000) Energy landscape of streptavidin-biotin complexes measured by atomic force microscopy. Biochemistry 39: 10219-10223.

Zhang X, Moy VT (2003) Cooperative adhesion of ligand receptor bonds. Biophys Chem 104: 271-278. 